\title{
Multidisciplinary decisions in breast cancer: does the patient receive what the team has recommended?
}

\author{
S Rajan ${ }^{*}$, , J Foreman ${ }^{2}$, M G Wallis ${ }^{3}$, C Caldas ${ }^{4}$ and P Britton ${ }^{5}$ \\ ${ }^{1}$ Cambridge Breast Unit, Box 97, Addenbrooke's Hospital, Hills Road, Cambridge, CB2 0QQ, UK; ${ }^{2} \mathrm{JCIS}$ University Department of \\ Oncology, NIHR Cambridge Biomedical Research Centre, Box 279, Addenbrooke's Hospital, Hills Road, CB2 0QQ, Cambridge, \\ UK; ${ }^{3}$ Cambridge Breast Unit, NIHR Cambridge Biomedical Research Centre, Box 97, Addenbrooke's Hospital, Hills Road, \\ Cambridge, CB2 0QQ, UK; ${ }^{4}$ Department of Oncology, University of Cambridge, Cambridge Breast Unit, NIHR Cambridge \\ Biomedical Research Centre, Experimental Cancer Medicine Centre, Cancer Research UK Cambridge Research Institute, Li Ka \\ Shing Centre, Robinson Way, Cambridge, CB2 ORE, UK and ${ }^{5}$ Cambridge Breast Unit, NIHR Cambridge Biomedical Research \\ Centre, Box 97, Addenbrooke's Hospital, Hills Road, Cambridge, CB2 0QQ, UK
}

Background: A multidisciplinary team (MDT) approach to breast cancer management is the gold standard. The aim is to evaluate MDT decision making in a modern breast unit.

Methods: All referrals to the breast MDT where breast cancer was diagnosed from 1 July 2009 to 30 June 2011 were included. Multidisciplinary team decisions were compared with subsequent patient management and classified as concordant or discordant.

Results: Over the study period, there were 3230 MDT decisions relating to 705 patients. Overall, $91.5 \%$ (2956 out of 3230) of decisions were concordant, 4.5\% (146 out of 3230), were discordant and 4\% (128 out of 3230) had no MDT decision. Of 146 discordant decisions, 26 (17.8\%) were considered 'unjustifiable' as there was no additional information available after the MDT to account for the change in management. The remaining 120 discordant MDT decisions were considered 'justifiable', as management was altered due to patient choice $(n=61)$, additional information available after MDT $(n=54)$ or MDT error $(n=5)$.

Conclusion: The vast majority of MDT decisions are implemented. Management alteration was most often due to patient choice or additional information available after the MDT. A minority of management alterations were 'unjustifiable' and the authors recommend that any patient whose treatment is subsequently changed should have MDT rediscussion prior to treatment.

A multidisciplinary team (MDT) approach to the management of breast cancer is the current gold standard in the United Kingdom (NHSBSP, 2005; Association of Breast Surgery at BASO, 2009; NICE, 2009; Willett et al, 2010). The implementation of MDT working was given impetus by the launch of the UK NHS breast screening programme in 1988 and national guidance on improving clinical outcomes for specific cancers, starting with breast cancer in 1996 (Cancer Guidance Sub-group of the Clinical Outcomes Group, 1996). The management decisions for breast cancer patients taken within the context of a MDT meeting involves collaboration between specialist teams and a consensus agreement being reached after consideration of available therapeutic options. This facilitates patient-centred, evidence-based treatment planning with good access to clinical trials (Shuster et al, 2000; Tripathy, 2003). This multidisciplinary approach to the management of patients with breast cancer is not unique to the United Kingdom and is widely utilised in other European countries, America, Australia and Asia (Chang et al, 2001; Zorbas et al, 2003; Saini et al, 2012).

Evaluating the impact of the introduction of MDT working is confounded by concurrent changes including increased specialisation of the cancer workforce, greater adherence to evidence-based 
guidelines and more effective treatments (Taylor et al, 2010). Although intuitively multidisciplinary care should be associated with better breast cancer survival, there have been relatively few publications to support this (Houssami and Sainsbury, 2006). A comparative cohort study assessed the survival of 13722 patients with breast cancer following formal introduction of MDT care by the Greater Glasgow Health Board in 1995 (Kesson et al, 2012). The non-intervention area, managed by the remaining health boards in the west of Scotland, continued to deliver care using traditional models. Before the introduction of MDT care, breast cancer mortality was $11 \%$ higher in the intervention area than in the non-intervention area with comparable all-cause mortality rates. After the introduction of MDT care in the intervention area, breast cancer mortality was $18 \%$ lower and all-cause mortality was $11 \%$ lower at 5 years than in the non-intervention area.

The assumption is that decisions on patient management taken at the MDT meeting are subsequently implemented. Early work in gastrointestinal MDT meetings demonstrated that up to $15 \%$ of MDT decisions may need revision after the meeting because key factors that influence the decision making process including patient's comorbidities had not been considered (Blazeby et al, 2006; Wood et al, 2008). More recently, an evaluation of the implementation of MDT decisions in patients with newly diagnosed benign and malignant breast disease reported the most common reason for a change in management was patient choice, with patients selecting an alternative treatment to that primarily recommended by the MDT (English et al, 2012).

The aim of this retrospective study is to evaluate the implementation of MDT decisions for patients with breast cancer in a modern breast unit. We will assess concordance between MDT decisions and subsequent patient management, and analyse the reasons for any discordant management. As far as the authors are aware, this is the largest study assessing the likelihood of breast cancer patients receiving the treatment decided at MDT meetings. In addition, we have evaluated the categories of MDT decisions that have not been documented to highlight which areas are most frequently overlooked to help improve documentation accuracy in the future.

\section{MATERIALS AND METHODS}

All referrals to the breast MDT where primary or recurrent breast cancer was diagnosed over a 2-year period from 1 July 2009 to 30 June 2011 were included in the study. This encompassed symptomatic patients referred by their family physician, screening referrals and second opinions from other hospitals. Patients were excluded if part of their treatment was subsequently carried out at another hospital whether privately or NHS. This study formed part of a registered audit at our institution, and did not require local ethical committee approval or patient consent.

On average, there are two MDT meetings per week with three radiologists, three oncologists, two breast surgeons, one pathologist, two specialist nurses, two breast care nurses, one trial nurse, one database manager and one MDT coordinator present. At each MDT meeting, all decisions are documented as care plans by the MDT coordinator and data are subsequently entered electronically into a dedicated breast database Joint Clinical Information System (JCIS). JCIS is an $n$-tier web-based clinical information system supported by a SQL server database. The system was built in-house in partnership with the breast clinical team using i5 web application and Microsoft technologies including Visual Basic. Joint Clinical Information System captures information throughout the patient's pathway from the first clinic visit, through to diagnosis and treatment (Britton et al, 2009). This was the primary system used to assess concordance of MDT decisions. Additional information was accessed from electronic medical records (EMR) that archives all clinic letters with access to full pathology and radiology reports. Imaging details not recorded on JCIS or EMR were accessed from the Radiology Information System.

The majority of patients are discussed at two MDT meetings: (i) an initial diagnostic MDT where the histological diagnosis is confirmed, neoadjuvant and surgical decisions are made, (ii) a second postoperative MDT where adjuvant treatment decisions are considered. At each of these MDT meetings, several decisions are made, broadly categorised into: (i) further needle biopsy, (ii) additional imaging including mammography, ultrasound, breast MRI, staging CT and bone scan, (iii) surgical decisions relating to breast and axillary management, (iv) oncology decisions pertaining to chemotherapy, endocrine therapy and radiotherapy. The authors recognise that all decisions made at the MDT are essentially recommendations of what is considered to be best practice for that patient, which they may accept or reject following an informed discussion with their clinician. However, for the purpose of clarity; the authors have classified each MDT decision into the following four groups:

(i) Definite MDT care plan. A definite MDT care plan is when the team has agreed on a single best action that is considered to be best practice for that patient. For example, a patient with a small unifocal invasive cancer and normal axillary ultrasound will have a specific MDT care plan that recommends a wide local excision (WLE) and sentinel lymph node biopsy (SLNB). All these MDT decisions were compared with subsequent patient management and classified as concordant or discordant.

(ii) MDT recommends one of two options. A proportion of patients were considered suitable for one of two possible options. For example, a patient who has histologically involved surgical margins following WLE may have a MDT care plan that recommends either re-excision of the involved margin or mastectomy. If either option was carried out, the decision was classified as concordant.

(iii) MDT suggests a flexible care plan. This includes patients who had flexible MDT care plans qualified by a variety of terms including 'discuss, possibly, query, borderline.' The outcome was classified as concordant if the MDT decision was implemented. If the MDT decision was not implemented after an informed discussion with the patient, this too was considered concordant but classified as 'considered but not implemented'. For example, the MDT may advise 'discuss chemotherapy' as the benefits are borderline; if the patient declines chemotherapy, this was classified as 'considered but not implemented' with patient choice as the reason for chemotherapy being declined. Patients within this flexible group who had a completely different treatment plan to that suggested by the MDT were classified as discordant. For example, a patient receiving primary endocrine therapy following a MDT decision to discuss surgery.

(iv) No MDT decision. This includes patients with no documented care plan relating to their surgical or oncological management - for example, if a patient underwent a SLNB but this decision had not been recorded in the MDT care plan.

Discordant decisions. A decision was considered discordant if the patient did not receive the management recommended by the MDT. All discordant decisions were investigated, and an evaluation made as to whether the change in management was 'justifiable' or 'unjustifiable'. A discordant decision was classified as 'justifiable' if this was the patient's choice or if there was additional information not available at the time of the MDT 
meeting. Additional information was classified into three main groups: (i) clinical information e.g., a patient's comorbidities or a surgeon finding a cancer to be clinically more advanced than initially thought, (ii) imaging information that increased locoregional extent or demonstrated metastatic disease (iii) pathology information e.g., hormone receptor status. A discordant decision was classified as 'unjustifiable' if there was no additional information available after the MDT meeting to account for the change in the patient's care plan.

\section{RESULTS}

Over the 2-year period, there were 3230 MDT decisions pertaining to the 705 patients (symptomatic $n=437$, screening $n=268$ ) included in the study. This equates to an average of five MDT decisions per patient made over two MDT meetings. Sixty-three patients were excluded, as part of their treatment was carried out at another hospital: privately $(n=53)$ or NHS $(n=10)$.

(i) Definite care plan. In $84.4 \%$ (2728 out of 3230 ) of decisions, there was a definite MDT care plan (Table 1). Of these definite MDT decisions, 2591 were concordant and 137 were discordant.

(ii) One of two options. In $2.6 \%$ (84 out of 3230) of decisions, the MDT felt it was reasonable to offer one of two options for the patient's management. All 84 decisions were concordant; one of two surgical options $n=47$, primary endocrine or primary surgery $n=19$, neoadjuvant chemotherapy or primary surgery $n=18$.

(iii) Flexible care plan. In 9\% (290 out of 3230) of decisions, the MDT suggested a flexible care plan qualified by terms such as 'discuss, query, possibly'. Of these flexible MDT care plans, 175 decisions were concordant and management plans were implemented, 106 decisions were 'considered but not implemented' and nine decisions were discordant. Of the 106 MDT decisions that were 'considered but not implemented,' $72.6 \%$ (77 out of 106) were declined by the patient following an informed discussion with the clinician. In the remaining $27.4 \%$ (29 out of 106) of MDT decisions that were 'considered but not implemented,' this was due to additional clinical information $(n=13)$, additional imaging information $(n=10)$ and MDT error $(n=6)$.

(iv) No MDT decision. Overall, 4\% (128 out of 3230) had no documented care plan relating to their surgical or oncological management. The two most common categories with no MDT decision were axillary surgery in 50\% (64 out of 128) and endocrine therapy in $20.3 \%$ (26 out of 128 ). In $14.8 \%$ (19 out of 128 ) of decisions, there was no breast surgical care plan; this was mainly patients who underwent neoadjuvant treatments that were

\begin{tabular}{|c|c|c|c|}
\hline $\begin{array}{l}\text { MDT decision } \\
\text { type }\end{array}$ & $\begin{array}{c}\text { Concordant, } \\
\mathbf{N}(\%)\end{array}$ & $\begin{array}{c}\text { Discordant, } \\
\mathbf{N}(\%)\end{array}$ & $\begin{array}{l}\text { Total } \mathbf{N}(\%) \\
\text { of decisions }\end{array}$ \\
\hline Definite care plan & 2591 (80.2\%) & $137(4.2 \%)$ & 2728 (84.4\%) \\
\hline One of two options & $84(2.6 \%)$ & $0(0 \%)$ & $84(2.6 \%)$ \\
\hline Flexible care plan & $281(8.7 \%)$ & $9(0.3 \%)$ & 290 (9\%) \\
\hline No MDT decision & N/A & N/A & $128(4 \%)$ \\
\hline Total & 2956 (91.5\%) & $146(4.5 \%)$ & 3230 (100\%) \\
\hline
\end{tabular}

not rediscussed at a MDT meeting prior to definitive surgery. In 9.4\% (12 out of 128), there was no MDT decision documented for radiotherapy and in 5.5\% (7 out of 128) for chemotherapy.

Overall, 91.5\% (2956 out of 3230) of MDT decisions were concordant and $4.5 \%$ (146 out of 3230) were discordant (Table 2). Of the 146 discordant MDT decisions, $82.2 \%$ (120 out of 146) were considered 'justifiable' and $17.8 \%$ (26 out of 146) were considered 'unjustifiable'.

Discordant MDT decisions - 'justifiable'. In 50.8\% (61 out of 120) of discordant 'justifiable' MDT decisions, the management plan was altered due to patient choice. In the remaining $49.2 \%$ (59 out of 120) of decisions, additional information was available after the MDT meeting that appropriately altered the initial management plan $(n=54)$ or a MDT error $(n=5)$ had been made. Additional information available after the MDT meeting included relevant clinical information $(n=34)$, imaging information that increased locoregional extent or demonstrated metastatic disease $(n=18)$ and pathology information $(n=2)$. In the group where additional information was available following the MDT meeting, $16.7 \%$ (9 out of 54) of discordant decisions were rediscussed at the next meeting. In all cases, the MDT was in agreement with the new management plan.

Discordant MDT decisions - 'unjustifiable'. In 17.8\% (26 out of 146) of discordant MDT decisions, the alteration in the care plan was considered 'unjustifiable' by the authors, as there was no additional information available after the MDT meeting to account for this change; this represents $0.8 \%$ of the total 3230 MDT decisions. In $53.9 \%$ (14 out of 26 ) of these decisions, the surgical management (breast surgery $n=6$, axillary surgery $n=8$ ) was altered (Table 3). In 27\% (7 out of 26) of decisions, imaging investigations (MRI $n=4$, ultrasound $n=2$, CT $n=1$ ) recommended at the MDT meeting were not performed. In 19.1\% (5 out of 26) of decisions, recommended oncology treatments (chemotherapy $n=3$, endocrine therapy $n=1$, radiotherapy $n=1$ ) were not prescribed.

Of the six discordant MDT decisions relating to breast surgery, two patients were upgraded from WLE to mastectomy at surgical review. In both patients, the size of the cancer exceeded $30 \mathrm{~mm}$ and may have been borderline for breast conservation once breast volume was taken into consideration. One patient was planned for a duct clearance on the basis of single-duct blood-stained nipple discharge and B3 diagnosis of atypical apocrine hyperplasia. Rather than undertaking a duct clearance, the surgeon requested further ultrasound-guided biopsies. This upgraded the preoperative diagnosis to apocrine DCIS allowing the patient to proceed to

Table 2. The number and proportion of concordant, discordant and no MDT decisions subdivided according to each of the main categories

\begin{tabular}{|l|c|c|c|c|}
\hline $\begin{array}{l}\text { Decision } \\
\text { category }\end{array}$ & $\begin{array}{c}\text { Concordant, } \\
\mathbf{N}(\%)\end{array}$ & $\begin{array}{c}\text { Discordant, } \\
\mathbf{N}(\%)\end{array}$ & $\begin{array}{c}\text { No MDT } \\
\text { decision, } \\
\mathbf{N}(\%)\end{array}$ & $\begin{array}{c}\text { Total } \\
\text { decisions }\end{array}$ \\
\hline Biopsy & $27(96.4 \%)$ & $1(3.6 \%)$ & $0(0 \%)$ & 28 \\
\hline Imaging & $230(92.4 \%)$ & $19(7.6 \%)$ & $0(0 \%)$ & 249 \\
\hline Breast surgery & $822(93.6 \%)$ & $37(4.2 \%)$ & $19(2.2 \%)$ & 878 \\
\hline Axillary surgery & $608(85.6 \%)$ & $38(5.4 \%)$ & $64(9 \%)$ & 710 \\
\hline Chemotherapy & $259(89 \%)$ & $25(8.6 \%)$ & $7(2.4 \%)$ & 291 \\
\hline Endocrine & $557(93.5 \%)$ & $13(2.2 \%)$ & $26(4.3 \%)$ & 596 \\
\hline Radiotherapy & $453(94.8 \%)$ & $13(2.7 \%)$ & $12(2.5 \%)$ & 478 \\
\hline Total & $2956(91.5 \%)$ & $146(4.5 \%)$ & $128(4 \%)$ & 3230 \\
\hline Abbreviation: MDT $=$ multidisciplinary team. &
\end{tabular}




\begin{tabular}{|c|c|c|}
\hline $\begin{array}{l}\text { Original MDT } \\
\text { decision }\end{array}$ & $\begin{array}{l}\text { Actual treatment } \\
\text { received }\end{array}$ & Comments \\
\hline WLE & $\mathrm{Mx}$ & $\begin{array}{l}38 \mathrm{~mm} \text { cancer (possibly } \\
\text { borderline for WLE) }\end{array}$ \\
\hline WLE & $M x$ & $\begin{array}{l}32 \mathrm{~mm} \text { cancer (possibly } \\
\text { borderline for WLE) }\end{array}$ \\
\hline Duct clearance & $\mathrm{Mx}$ & $\begin{array}{l}\text { Single stage therapeutic breast } \\
\text { surgery }\end{array}$ \\
\hline $\mathrm{Mx}$ & WLE & $27 \mathrm{~mm}$ cancer with clear margins \\
\hline$M x$ & $\begin{array}{l}\text { WLE then cavity } \\
\text { shave }\end{array}$ & $\begin{array}{l}2 \text { operations to achieve clear } \\
\text { margins }\end{array}$ \\
\hline$M x^{a}$ & Primary endocrine & Not reassessed for surgery \\
\hline ALND $^{a}$ & Primary endocrine & Not reassessed for surgery \\
\hline ALND & SLNB then ALND & $\begin{array}{l}\text { 2-stage axillary surgery (positive } \\
\text { SLNB) }\end{array}$ \\
\hline SLNB & ALND & Positive ALND (4/20 nodes) \\
\hline SLNB & ALND & Positive ALND (1/14 nodes) \\
\hline SLNB & ALND & Negative ALND (0/8 nodes) \\
\hline SLNB & ALND & Negative ALND (0/8 nodes) \\
\hline SLNB & ALND & Negative ALND ( $0 / 40$ nodes) \\
\hline SLNB & No axillary surgery & $\begin{array}{l}\text { DCIS only with no invasive } \\
\text { breast cancer }\end{array}$ \\
\hline \multicolumn{3}{|c|}{$\begin{array}{l}\text { Abbreviations: } A L N D=\text { axillary lymph node dissection; } D C I S=\text { ductal carcinoma in situ; } \\
M D T=\text { multidisciplinary team; } M x=\text { mastectomy; } S L N B=\text { sentinel lymph node biopsy; } \\
\text { WLE = wide local excision. } \\
a_{\text {represents the same patient. }}\end{array}$} \\
\hline
\end{tabular}

single-stage therapeutic surgery. Two patients originally planned for mastectomy were considered suitable for WLE at surgical review. The first patient had a successful WLE and the second patient required two operations to achieve clear margins. One patient planned for mastectomy and axillary lymph node dissection (ALND) was started on neoadjuvant endocrine treatment and has not subsequently undergone surgery.

Of the remaining seven discordant MDT decisions relating to axillary surgery, one patient planned for ALND was considered suitable for SLNB by the surgical team. The MDT recommended ALND in light of a $90 \mathrm{~mm}$ grade 2 cancer, but the surgeon felt SLNB was a suitable option as the axillary ultrasound was normal. The SLNB was positive and the patient required a completion ALND. Five patients planned for SLNB were upgraded to ALND by the surgeon; two patients had a positive ALND and three patients had a negative ALND. One patient planned for SLNB did not undergo any axillary surgery.

MDT error. In $0.3 \%$ (11 out of 3230) of decisions, an error had been made at the time of MDT discussion or documentation. Following the MDT meeting, the clinician detected the error and appropriately altered the original MDT care plan. In $54.5 \%$ (6 out of 11) of decisions, a MDT error was detected by the oncologist when reassessing the patient's potential benefit from adjuvant chemotherapy or radiotherapy and finding this to below the unit's threshold for recommendation or discussion. In three patients, the MDT advised inappropriate or unnecessary imaging investigations including a breast MRI in a pregnant patient. In two patients, the MDT care plan mistakenly recommended SLNB despite a preoperative malignant axillary node core biopsy result.

\section{DISCUSSION}

Multidisciplinary care is central to the delivery of a high-quality service. Over the 2-year study period, there were 3230 MDT decisions relating to 705 patients. Overall, $91.5 \%$ of decisions were concordant with patients receiving the management advised by the MDT and $4.5 \%$ of decisions were not implemented. This is comparable to recent work evaluating the implementation of MDT decisions in 210 patients with newly diagnosed benign and malignant breast disease, in which $6.9 \%$ of 289 decisions were not implemented over a 3-month period (English et al, 2012).

Discordant MDT decisions - 'justifiable'. The strength of a MDT decision relies heavily on the clinical information available at the time of discussion. Approximately $50 \%$ of discordant MDT decisions were altered due to additional information that became available after the meeting. In $63 \%$ (34 out of 54) of these decisions, there was additional clinical information (surgical information $n=22$, patient's comorbidities $n=12$ ) that appropriately altered the original MDT care plan. This is comparable to early work evaluating the quality of decision making in gastrointestinal MDT meetings, where the most common reason for alteration of MDT decisions after the meeting was due to additional relevant clinical information such as patient's comorbidities (Blazeby et al, 2006; Wood et al, 2008). In addition, a more recent prospective study evaluating consecutive upper gastrointestinal MDT decisions demonstrated that when the MDT recommendation was not implemented, there was a significant delay to finally starting treatment (Goolam-Hossen et al, 2011).

Implementing a policy of clinical review by a surgeon at the time of initial assessment of patients who are likely to have a borderline conservable cancer on imaging and clinical grounds may enable more reliable and specific subsequent MDT decision making. A more quantitative assessment of a patient's general health and relevant comorbidities with a performance status score documented at the time of initial assessment might also help inform MDT decisions. In this study, $16.7 \%$ of discordant 'justifiable' MDT decisions were rediscussed when additional information became available. We have now introduced a policy that all discordant decisions should be discussed at a subsequent MDT meeting prior to alteration of the care plan.

Discordant MDT decisions - 'unjustifiable'. In 0.8\% (26 out of 3230) of all MDT decisions, there was no additional information available after the meeting to account for the change in management. The most common decision to be altered was surgical management, accounting for $53.9 \%$ (14 out of 26) of the discordant decisions. With regards to breast surgical decisions made at the MDT meeting, breast imaging provides a guide in assessing the feasibility of breast conservation. When breast surgical intent is altered $(n=6)$, this is often in patients with borderline suitability for breast conservation. With regards to alteration in axillary surgical intent $(n=8)$, there were five patients that proceeded straight to ALND despite a normal axillary ultrasound. These decisions were usually taken in patients with larger, more aggressive tumours where the surgeon presumably felt that lymph node involvement was highly likely and was trying to reduce the need for two axillary operations. Yet, $60 \%$ (3 out of 5) of these patients proved to be negative at ALND. A risk stratified protocol should be developed based upon patient age, overall health status and likelihood of nodal involvement to enable more uniform patient care.

Patient choice. Patient choice factors heavily in the final management pathway chosen. When definite MDT decisions are recommended, approximately $50 \%$ of 'justifiable' discordant decisions are due to treatment being declined by the patient. When the MDT proposes a flexible care plan, 26.6\% (77 out of 290) of 


\begin{tabular}{|c|c|c|c|}
\hline $\begin{array}{l}\text { MDT decision } \\
\text { category }\end{array}$ & $\begin{array}{l}\text { Decisions } \\
\text { declined }\end{array}$ & $\begin{array}{l}\text { Total flexible } \\
\text { decisions }\end{array}$ & $\begin{array}{c}\% \text { of decisions } \\
\text { declined }\end{array}$ \\
\hline Biopsy & 1 & 10 & 10 \\
\hline Imaging & 0 & 8 & 0 \\
\hline Breast surgery & 30 & 127 & 23.6 \\
\hline Axillary surgery & 0 & 27 & 0 \\
\hline Chemotherapy & 29 & 61 & 47.5 \\
\hline Endocrine & 16 & 46 & 34.8 \\
\hline Radiotherapy & 1 & 11 & 9.1 \\
\hline Total & 77 & 290 & 26.6 \\
\hline
\end{tabular}

management decisions are declined by the patient (Table 4). Following an MDT decision to 'discuss' adjuvant treatments, usually when the treatment benefits are borderline, $47.5 \%$ of patients declined chemotherapy and $34.8 \%$ of patients declined endocrine therapy. This is comparable to recently published data that confirms that patient choice was the most common reason for a change in the management plan, and MDT decisions were significantly more likely to change in older than younger women and in those with finally proven malignant rather than benign breast disease (English et al, 2012).

The factors affecting multidisciplinary decision making are complex. Qualitative data highlight that MDT discussions are often dominated by those with surgical, medical or diagnostic expertise, with limited contributions from those with a nursing, palliative or psychosocial background who may have relevant information about patient-centred issues (Lanceley et al, 2008; Lamb et al, 2011). This highlights the importance of the role of the breast care nurse in conveying the patient's initial views in the MDT meeting, as well as having a full and open discussion with the patient prior to making any treatment decisions (National Cancer Action Team, 2010).

MDT error. In $0.3 \%$ (11 out of 3230) of all MDT decisions, an error had been made at the time of the MDT discussion or documentation. The clinician detected the error at the time of clinical consultation and appropriately altered the MDT care plan. In many units, clinicians use prognostication and treatment benefit tools such as PREDICT or Adjuvant! Online to help make informed decisions about treatment. More than 50\% of MDT errors are related to mistakes in the calculation of potential benefit of adjuvant treatments during the meeting. This could result in a patient inappropriately being prescribed chemotherapy despite falling short of the unit's threshold for recommendation of treatment. Any MDT errors not discovered in a subsequent clinic will not be detected on the basis of this study. Although it is likely that these errors are uncommon, we have not formally reassessed all of the concordant MDT decisions, and so the number of MDT errors may well be higher.

Accuracy of MDT documentation. Overall, 4\% (128 out of 3230) had no documented MDT decision relating to their surgical or oncological management. The two most common categories with 'no MDT decision' were axillary surgery in 50\% (64 out of 128) and endocrine therapy in $20.3 \%$ (26 out of 128). It is difficult to be certain what proportion of these decisions were discussed but not documented in the MDT care plan or how many were overlooked and the responsible clinician subsequently took a decision independently. The previous study evaluating the implementation of MDT decisions in patients with breast disease excluded patients where the MDT decision was not recorded (English et al, 2012). This study is the first to evaluate the specific categories of MDT decisions that have not been documented in order to highlight which areas are most frequently overlooked and consider ways of improving the accuracy of documentation. A useful solution would be for the MDT coordinator to enter data electronically while the meeting is in progress, and this could be projected live on screen, helping to improve documentation accuracy and reduce potential for error. A template with dropdown menus for each of the core categories (imaging, surgery and oncology) would help to improve transparency of MDT decisions, increase clarity of treatment intent and reduce the plethora of potentially ambiguous terms currently used.

In summary, this single unit study demonstrates that the majority of breast cancer patients do receive the treatment decided at the MDT meeting. If management decisions are altered, it is most often due to patient choice or additional information becoming available after the MDT. This study highlights the importance of patient choice in decision making and the role of the breast care nurse in the MDT setting who is able to convey the patient's preferences, therefore facilitating individualised treatment pathways. The more information available at the time of the MDT discussion, the more robust the decision making process will be. A more detailed initial clinical assessment of likely tumour extent by an experienced clinician, preferably a surgeon and a quantifiable record of the patient's general health would aid subsequent MDT management decisions and reduce the number of discordant decisions. We have now introduced a policy of rediscussing all discordant decisions at a subsequent MDT meeting prior to finalising the new management plan. There is room for improvement in MDT documentation and the live entry of data using a template with dropdown options would ensure all elements of treatment are covered and reduce the potential for errors.

\section{ACKNOWLEDGEMENTS}

The authors were supported with research funding from the National Institute for Health Research (NIHR) Cambridge Biomedical Research Centre (CBRC) and Experimental Cancer Medicine Centre. The JCIS team are funded by Cancer Research UK and NIHR CBRC.

\section{CONFLICT OF INTEREST}

The authors declare no conflict of interest.

\section{REFERENCES}

Association of Breast Surgery at BASO (2009) Surgical guidelines for the management of breast cancer. Eur J Surg Oncol 35: S1-S22.

Blazeby JM, Wilson L, Metcalfe C, Nicklin J, English R, Donovan JL (2006) Analysis of clinical decision-making in multi-disciplinary cancer teams. Ann Oncol 17: 457-460.

Britton P, Duffy SW, Sinnatamby R, Wallis MG, Barter S, Gaskarth M, O'Neill A, Caldas C, Brenton JD, Forouhi P, Wishart GC (2009) One-stop diagnostic breast clinics: how often are breast cancers missed? Br J Cancer 100: $1873-1878$.

Cancer Guidance Sub-group of the Clinical Outcomes Group (1996) Improving outcomes in breast cancer. Department of Health: London.

Chang JH, Vines E, Bertsch H, Fraker DL, Czerniecki BJ, Rosato EF, Lawton T, Conant EF, Orel SG, Schuchter L, Fox KR, Zieber N, Glick JH, Solin LJ (2001) The impact of a multidisciplinary breast cancer center on 
recommendations for patient management: the University of Pennsylvania experience. Cancer 91: 1231-1237.

English R, Metcalfe C, Day J, Rayter Z, Blazeby JM (2012) A prospective analysis of implementation of multi-disciplinary team decisions in breast cancer. Breast J 18: 459-463.

Goolam-Hossen T, Metcalfe C, Cameron A, Rocos B, Falk S, Blazeby JM (2011) Waiting times for cancer treatment: the impact of multidisciplinary team meetings. Behav Inform Technol 30: 467-471.

Houssami N, Sainsbury R (2006) Breast cancer: multidisciplinary care and clinical outcomes. Eur J Cancer 42: 2480-2491.

Kesson EM, Allardice GM, George WD, Burns HJG, Morrison DS (2012) Effects of multidisciplinary team working on breast cancer survival: retrospective, comparative, interventional cohort study of 13722 women. BMJ 344: e2718.

Lamb BW, Brown KF, Nagpal K, Vincent C, Green JS, Sevdalis N (2011) Quality of care management decisions by multidisciplinary cancer teams: a systematic review. Ann Surg Oncol 18: 2116-2125.

Lanceley A, Savage J, Menon U, Jacobs I (2008) Influences on multidisciplinary team decision-making. Int J Gynecol Cancer 18: 215-222.

National Cancer Action Team (2010) The characteristics of an effective multidisciplinary team. Accessed from ncat.nhs.uk.

NHSBSP (2005) Quality assurance guidelines for breast cancer screening radiology. NHS Cancer Screening Programmes, Publication No. 59. Accessed from www.cancerscreening.nhs.uk.

NICE (2009) Early and locally advanced breast cancer: diagnosis and treatment. Clinical guideline 80. Accessed from www.nice.org.uk.

Saini KS, Taylor C, Ramirez AJ, Palmieri C, Gunnarsson U, Schmoll HJ, Dolci SM, Ghenne C, Metzger-Filho O, Skrzypski M, Paesmans M,
Ameye L, Piccart-Gebhart MJ, De Azambuja E (2012) Role of the multidisciplinary team in breast cancer management: results from a large international survey involving 39 countries. Ann Oncol 23: 853-859.

Shuster TD, Girshovich L, Whitney TM, Hughes KS (2000) Multidisciplinary care for patients with breast cancer. Surg Clin North Am 80: 505-533.

Taylor C, Munro AJ, Glynne-Jones R, Griffith C, Trevatt P, Richards M, Ramirez AJ (2010) Multidisciplinary team working in cancer: what is the evidence? BMJ 340: 743-745.

Tripathy D (2003) Multidisciplinary care for breast cancer: barriers and solutions. Breast J 9: 60-63.

Willett AM, Michell MJ, Lee MJR (2010) Best practice diagnostic guidelines for patients presenting with breast symptoms. Department of Health, Gateway reference 13737. Accessed from www.dh.gov.uk/publications.

Wood JJ, Metcalfe C, Paes A, Sylvester P, Durdey P, Thomas MG, Blazeby JM. (2008) An evaluation of treatment decisions at a colorectal cancer multidisciplinary team. Colorectal Dis 10: 769-772.

Zorbas H, Rainbird K, Luxford K, Barraclough B, Redman S (2003) Multidisciplinary care for women with early breast cancer in the Australian context: what does it mean? Med J Aust 179: 528-531.

This work is published under the standard license to publish agreement. After 12 months the work will become freely available and the license terms will switch to a Creative Commons AttributionNonCommercial-Share Alike 3.0 Unported License. 